\title{
Développement précoce de la souris : divergence des deux premiers lignages
}

Le développement précoce de la souris correspond à la période allant de la fécondation à la formation du blastocyste, juste avant implantation dans la paroi utérine. Le blastocyste est constitué de deux types cellulaires, la masse cellulaire interne et le trophectoderme. La caractéristique principale du développement précoce est qu'il existe une grande flexibilité dans les voies de différenciation de ces deux premiers lignages. En effet, pendant cette période, les cellules restent totipotentes et peuvent donner des dérivés appartenant à l'un ou l'autre des deux lignages. La ségrégation cellulaire dépend des interactions cellulaires et de la géométrie des cellules, contrôlée au niveau cytoplasmique [1, 2].

C'est C.H. Waddington qui, en 1940, a introduit la notion de " paysage épigénétique ". Pour lui, la cellule possède dans son patrimoine génétique toutes les informations nécessaires à plusieurs types de développement, mais sous l'influence de facteurs externes (milieu environnant, contacts intercellulaires...) une seule voie de détermination est choisie. Cette définition du "paysage épigénétique " est certes un peu restreinte puisqu'on assiste souvent à "un retour " vers une autre voie de détermination, déjà discriminée a priori d'après un tel schéma (lors d'un phénomène de métaplasie une cellule totalement différenciée se dédifférencie puis se redifférencie : cela a lieu par exemple chez le triton où les cellules de l'iris dorsal peuvent reformer un cristallin). On peut tout de même en déduire une définition du terme " épigénétique ", correspondant à une information qui n'est pas codée directement par le génome et qui

tir de cette définition nous allons donc essayer de déterminer la nature et l'importance des événements épigénétiques dans le développement précoce de la souris.

L'œuf de souris est bloqué en métaphase de seconde division de méiose lors de l'ovulation. Après fécondation, on observe une reprise de la méiose et l'émission du second globule polaire. Lors de la segmentation, les cycles cellulaires des blastomères ne sont pas synchrones et les plans de clivage n'ont pas d'orientation particulière. Au stade 2 cellules, il $y$ a activation du génome embryonnaire. Le stade 8 cellules est une étape charnière du développement précoce : c'est à ce stade que la morula se contracte, par aplatissement des cellules les unes sur les autres (phénomène de " compaction "). C'est à partir du stade 16 qu'apparaît une régionalisation de l'embryon en deux populations cellulaires distinctes :

- les cellules internes, précurseurs de la masse cellulaire interne ;

- les cellules externes, à l'origine du trophectoderme et d'une partie de la masse cellulaire interne.

A partir du stade 32 cellules, les cellules externes constituent un épithélium véritable à l'origine de la formation de la cavité du blastocyste, ou blastocœle. Au stade blastocyste, les cellules externes du trophectoderme seront à l'origine de la plupart des annexes embryonnaires. Les cellules de la masse cellulaire interne formeront le reste des annexes et l'embryon lui-même (figure 1).

L'étude de la nature des interactions cellulaires et de leur influence sur le devenir des blastomères a été plus particulièrement approfondie par manipulation d'embryons de souris in vitro: par exemple, les blastomères peuvent être isolés et placés dans des contextes cellulaires différents. Normalement, les cellules externes du blastocyste peuvent se différencier soit en cellules internes, soit en cellules du trophectoderme, les cellules internes ne peuvent se différencier qu'en cellules de la masse cellulaire interne [3]. Toutefois, si expérimentalement, on place une cellule interne à l'extérieur de l'embryon, elle va se différencier en cellule externe et donnera du trophectoderme [4].

Des facteurs épigénétiques vont intervenir dans le contrôle de ce choix binaire. En particulier, l'existence de divisions cellulaires asymétriques, entre le stade 8 et le stade 16 (figure 2), est à l'origine de la mise en place de ces deux lignages. Ces divisions asymétriques reposent sur l'apparition, à la compaction, d'une asymétrie de l'organisation cellulaire. En effet, toutes les cellules développent à ce stade une polarité à la fois corticale et cytoplasmique définissant un axe apical/basal. Au stade 16, après division, seules les cellules externes conservent cette polarité tandis que les cellules internes sont apolaires.

La polarisation des blastomères se met en place lors de la compaction au stade 8 cellules. Il se produit une redistribution apicale d'organites cytoplasmiques (endosomes et éléments du cytosquelette), puis des microvillosités se développent à la surface de la cellule. Intuitivement on peut penser que la polarité cytoplasmique induit la mise en place de la polarité corticale puisqu'elle précède temporellement cette dernière. Ce n'est pas toujours le cas : la polarité corticale peut se mettre en place en l'absence de polarité cytoplasmique. 


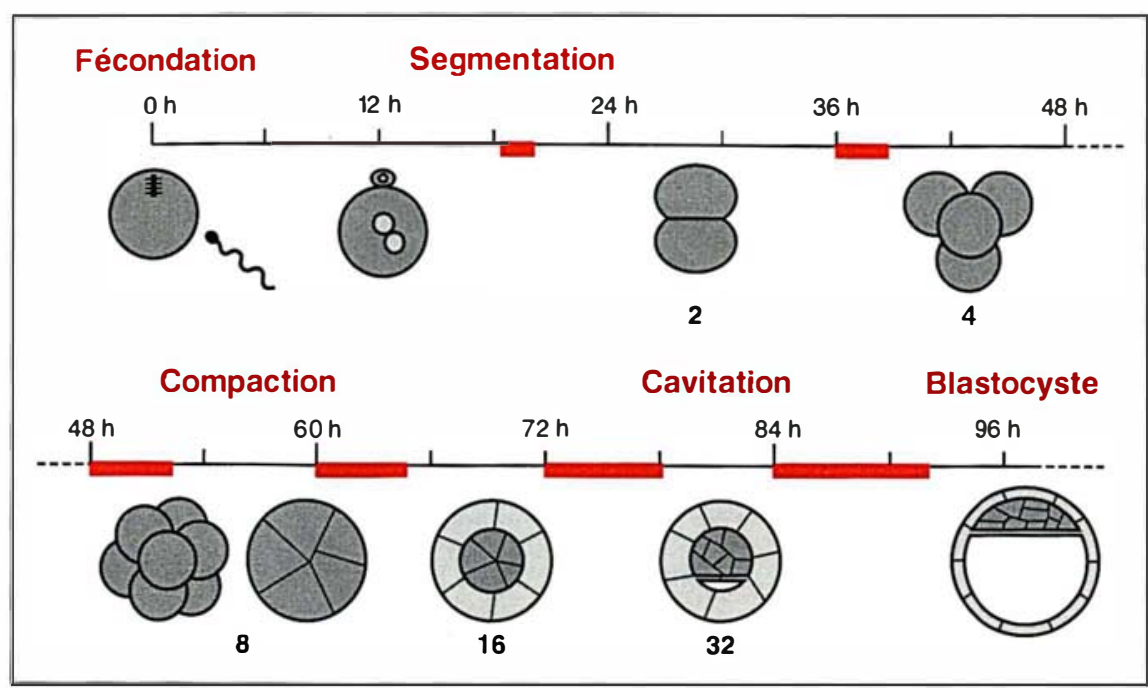

Figure 1. Développement de l'embryon de souris avant implantation. Le nombre de cellules présentes dans l'embryon à chaque stade est indiqué. $A$ partir du stade 16, les cellules externes sont représentées avec un ombrage plus clair que les cellules internes. Sur l'échelle de temps, les rectangles rouges représentent les périodes durant lesquelles une ou plusieurs cellules sont en mitose.

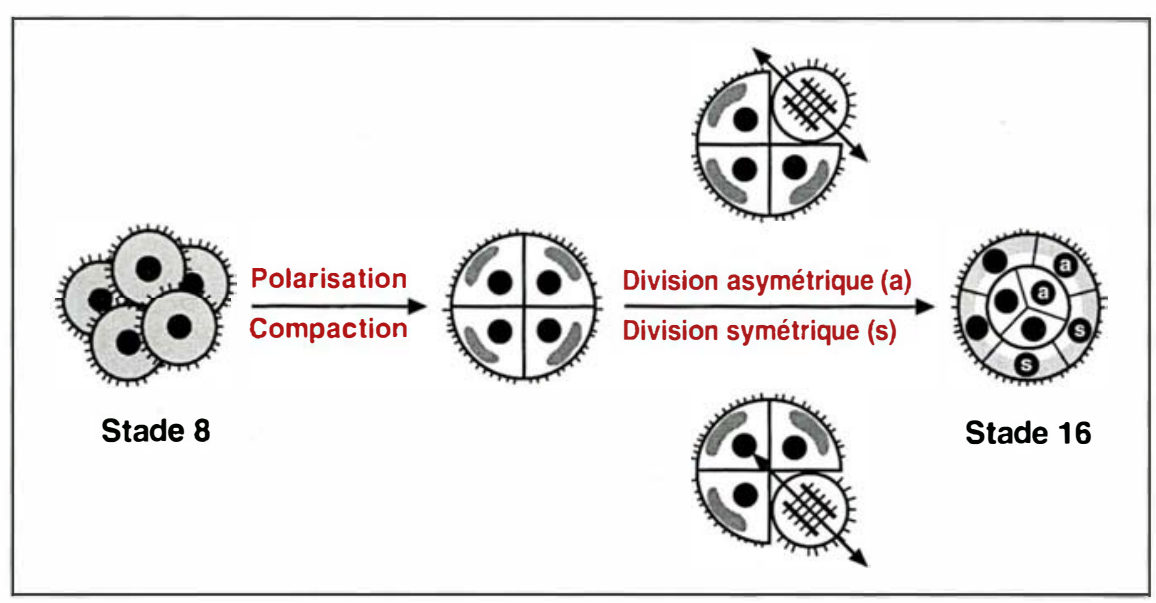

Figure 2. Mécanismes cytoplasmiques impliqués dans la divergence des deux premiers lignages de l'embryon. Polarisation : lors de la compaction, les blastomères, jusqu'alors symétriques, se polarisent (formation d'un pôle apical comprenant des microvillosités à la surface et divers organites dans le cytoplasmel. Division asymétrique : lors de la division cellulaire, l'orientation du fuseau mitotique, parallele ou orthogonal à l'axe de polarité de la cellule, détermine le phénotype des cellules filles : deux cellules polarisées si le fuseau est orthogonal (division symétrique) ou une cellule polarisée et une cellule non polarisée s'il est parallèle (division asymétrique). Seules les deux situations extrêmes sont représentées, le fuseau pouvant avoir n'importe quelle orientation par rapport à l'axe de polarité de la cellule. Adhérence : les cellules non polarisées sont adhérentes sur toute leur surface alors que les cellules polarisées ne le sont que dans la région baso-latérale, la partie apicale n'étant pas adhérente. De ce fait, les cellules polarisées restent à l'extérieur de l'embryon et entourent les cellules non polarisées.

$\mathrm{m} / \mathrm{s} n^{\circ} 9$, vol. 7, novembre 91
L'aplatissement intercellulaire est lié à l'interaction des molécules d'uvomoruline (molécule d'adhérence intercellulaire dépendante du calcium, ou E-cadhérine) entre les membranes basolatérales des blastomères. Plus tard, l'uvomoruline est répartie uniformément à la surface des cellules internes et exclusivement sur la surface basolatérale des cellules externes [5]. La redistribution des microvillosités au pôle apical des cellules à ce stade n'est pas toujours consécutive à une extension des contacts intercellulaires : des pôles de microvillosités peuvent se former dans des embryons dont la compaction a été empêchée artificiellement [6]. De plus, les cellules polarisées isolées gardent leur pôle de microvillosités en l'absence de contact avec d'autres cellules. Les travaux réalisés sur des paires de blastomères isolés ont permis de montrer qu'en l'absence de compaction, les axes de polarité développés dans les cellules prennent des orientations tout à fait aléatoires (l'axe de polarité dans ces paires de cellules étant normalement orienté perpendiculairement à la zone de contact). La compaction permettrait une synchronisation et une orientation homogène des axes de polarité au sein de l'embryon.

L'importance des contacts cellulaires dans l'acquisition du phénotype ainsi que sa rapidité de mise en place suggèrent l'implication dans ce mécanisme de structures capables à la fois de détecter des contacts intercellulaires et de modifier l'organisation cellulaire : c'est là qu'interviennent les éléments du cytosquelette. Au stade 8 , le réseau microtubulaire change d'organisation : il se produit une redistribution des microtubules vers le pôle apical des blastomères. La disparition des microtubules les plus labiles de la partie basale des cellules semble permettre l'apparition de jonctions communicantes et favoriser l'adhérence intercellulaire, tandis que leur enrichissement dans la partie apicale stabiliserait le pôle de microvillosités et d'organites cytoplasmiques [2]. Les microtubules semblent donc coordonner les différents changements ayant lieu lors de la compaction et renforcer l'asymétrie établie dans le cortex cellulaire. Les 
microfilaments d'actine, quant à eux, semblent être impliqués dans des événements terminaux de polarisation plutôt que dans la coordination entre aplatissement et polarisation. Tous les changements qui se produisent à la compaction semblent être déclenchés par des mécanismes posttraductionnels tels que des phosphorylations impliquant, entre autres, la voie de la protéine kinase C [7]. Il reste à déterminer clairement la nature des signaux échangés entre les cellules de l'embryon et l'interaction de ces derniers avec des éléments du cytosquelette susceptibles de les interpréter, tels que les microtubules. Au cours des divisions cellulaires, la polarité corticale reste stable tandis que la polarité cytoplasmique, peutêtre plus dépendante des éléments du cytosquelette, disparaît puis se reforme dans les cellules filles polarisées qui ont hérité tout ou partie du pôle de microvillosité [8].

-A partir du stade 8, l'orientation du fuseau mitotique par rapport à l'axe de polarité de la cellule mère va déterminer le phénotype des deux cellules filles. Schématiquement, si l'orientation du fuseau de division coïncide avec l'axe de polarité de la cellule mère une des deux cellules filles sera polarisée (division " asymétrique "), s'il lui est perpendiculaire les deux cellules filles seront polarisées (division "symétrique ") (figure 2). La position, interne ou externe, occupée par ces dernières va d'ailleurs dépendre de leur phénotype : cellules polarisées en périphérie et cellules non polarisées à l'intérieur de l'embryon. En effet, les cellules non polarisées sont adhérentes sur toute la surface tandis que les cellules polarisées ne le sont que sur leur surface basolatérale : ces dernières ont donc tendance à englober les cellules non polarisées.

L'orientation du fuseau mitotique au stade 8 se faisant au hasard, le rapport entre le nombre de cellules internes et externes est très variable au stade 16. Dans les blastocystes cette variabilité est beaucoup plus faible, parce que des mécanismes de compensation liés à la géométrie des cellules existent $[2,3,9]$. Le volume de l'embryon étant constant, plus le nombre de cellules augmente, plus leur taille diminue et plus les orientations possibles du fuseau sont restreintes :

- si il y a peu de cellules internes, les cellules externes seront pyramidales, les fuseaux parallèles à l'axe de polarité et les divisions asymétriques, ce qui augmentera la proportion de cellules internes au stade suivant; - si il y a beaucoup de cellules internes, les cellules externes seront aplaties, les fuseaux parallèles à la surface de l'embryon et les divisions symétriques, ce qui ne modifiera pas la proportion de cellules internes.

Ces mécanismes dépendant de l'organisation et de la géométrie des cellules embryonnaires précoces influent donc sur le programme génétique de différenciation. Par exemple, certaines cytokératines semblent caractéristiques du trophectoderme. En fait, les premiers filaments intermédiaires de kératine apparaissent au stade 8, dans quelques cellules. Ils se répartissent au stade 16 de façon homogène entre cellules externes et internes et c'est seulement à partir du stade 32 que les cytokératines se répartissent préférentiellement dans les cellules externes et disparaissent progressivement des cellules internes [10]. La régulation négative de l'expression génétique des cytokératines est en fait fonction de la position des cellules dans l'embryon. D'autres marqueurs semblent être contrôlés positivement, ils s'expriment uniquement dans les cellules externes à partir du stade 16 .

Il apparaît donc que la géométrie des cellules (à la fois pour ce qui concerne leur organisation et leurs contacts) joue un rôle important dans la détermination des cellules embryonnaires précoces de la souris. Enfin, la voie de différenciation empruntée par ces cellules n'est pas irréversible, des mécanismes épigénétiques pouvant modifier leur destin

\section{Marie-Hélène Verlhac}

Bernard Maro

Institut Jacques-Monod, Cnrs, Université Paris 7, Tour 43, 2, place Jussieu, 75005 Paris, France.

\section{RÉFÉRENCES}

1. Fleming TP, Johnson MH. From egg to epithelium. Ann Rev Cell Biol 1988; 4: 459-85.

2. Maro B, Gueth-Hallonet C, Aghion J, Antony C. Cell polarity and microtubule organisation during mouse early embryogenesis. Development $1991 ; 17-25$.

3. Fleming TP. A quantitative analysis of cell allocation to trophectoderm and inner cell mass in the mouse blastocyst. Dev Biol 1987 ; 119 : 520-31.

4. Ziomek CA, Johnson MH. The roles of phenotype and position in guiding the fate of 16-cell mouse blastomeres. Dev Biol $1982 ; 91$ : 440-7

5. Vestweber D, Gossler A, Boller K, Kemler R. Expression and distribution of cell adhesion molecule uvomorulin in mouse preimplantation embryos. Dev Biol 1987 ; $124: 451-6$.

6. Houliston E, Pickering SJ, Maro B. Alternative routes for the establishment of surface polarity during compaction of the mouse embryo. Dev Biol 1989; 134 : 342-50.

7. Winkel GK, Ferguson JE, Takeichi M, Nucitelli M. Activation of protein kinase $C$ triggers premature compaction in the fourcell stage mouse embryo. Dev Biol 1990 ; 138 : $1-15$

8. Johnson MH, Ziomek CA. The foundation of two distinct cell lineages within the mouse morula. Cell 1981; 24 : 71-80.

9. Johnson MH, Ziomek CA. Cell interactions influence the fate of blastomeres undergoing the transition from the 16- to the 32-cell stage. Dev Biol 1983 ; 95 : 211-8. 10. Chisholm JC, Houliston E. Cytokeratin assembly in the mouse preimplantation embryo. Development 1987 ; 101 : 565-82.

AVIS AUX AUTEURS

\section{DE TRAVAUX IMPORTANTS}

$\mathrm{m} / \mathrm{s}$ propose aux auteurs de travaux importants, publiés dans des revues d'audience internationalc et de premier niveau, de présenter leurs résultats sous forme de brève, de nouvelle, voire de mini-synthèse, au mieux publiés dans médecine/sciences, parallèlement à l'article princeps.

\section{LA RÉDACTION}

Les manuscrits doivent être adressés à médecine/sciences, 6, rue Blanche, 92120 Montrouge, France.

Tél. : (1) 47.35.85.52

Fax : 46.57.10.09. 